\title{
The genus Andraca (Lepidoptera, Endromidae) in China with descriptions of a new species
}

\author{
Xing Wang ${ }^{1, \dagger}$, Ling Zeng ${ }^{2, \ddagger}$, Min Wang ${ }^{2, \S}$
}

I Department of Entomology, South China Agricultural University, Guangzhou, Guangdong 510640, P.R. China. Present address: Institute of Entomology, College of Biosafety Science and Technology, Hunan Agricultural University, Changsha 410128 Hunan, China; and Provincial Key Laboratory for Biology and Control of Plant Diseases and Insect Pests, Changsha 410128, Hunan, China 2 Department of Entomology, South China Agricultural University, Guangzhou, Guangdong 510640, P.R. China

† urn:lsid:zoobank.org:author:F8727887-0014-42D4-BA68-21B3009E8C7F

† urn:lsid:zoobank.org:author:7981BFOE-D1F8-43CA-A505-72DBBA140023

§ urn:lsid:zoobank.org:author:D683614E-1F58-4CA8-9D80-B23BD41947A2

Corresponding author: Ling Zeng (zengling@scau.edu.cn)

Academic editor: N. Wahlberg| Received 21 January 2011 | Accepted 15 August 2011 | Published 8 September 2011

urn:lsid:zoobank.org:pub:33D4BBFB-4B7D-4BBC-B34C-17D2E7F99F67

Citation: Wang X, Zeng L, Wang M (2011) The genus Andraca (Lepidoptera, Endromidae) in China with descriptions of a new species. ZooKeys 127: 29-42. doi: 10.3897/zookeys.127.928

\begin{abstract}
The six species of the genus Andraca Walker hitherto known from China are reviewed, and a new species, A. gongshanensis, sp. n., described from Yunnan Province, China. Adults and male genitalia of all examined species are illustrated, together with a distributional map. A key to all seven Chinese Andraca species is provided. The types of the new species are deposited in SCAU (South China Agricultural University, Guangzhou, China) and HUNAU (Hunan Agricultural University, Changsha, China).
\end{abstract}

\section{Keywords}

Taxonomy, Lepidoptera, Endromidae, Andraca, new species, China 


\section{Introduction}

The genus Andraca was established by Walker (1865) with Andraca bipunctata Walker 1865 as its type-species, a species well known as one of the most serious pests of tea plants (Chu and Wang 1996). It was placed in family Bombycidae for over 150 years, but was recently transferred to family Endromidae based on the molecular study of Zwick et al. (2011). Kishida (1993) reported A. theae and A. olivacea from Taiwan. Chu and Wang (1993) recorded three Andraca species from China: A. bipunctata is widely distributed in central and southern China, A. henosa Chu \& Wang, 1993 was listed from Yunnan, and $A$. hedra Chu \& Wang, 1993 from Hainan and Fujian; in this paper, they also included Andraca gracilis Butler 1885, which is currently placed in the genus Pseudandraca Miyata, 1970. Yang (1995) added one species, A. flavamaculata Yang, 1995, to the Chinese Andraca fauna. Owada et al. (2002) reviewed three species of Andraca from Vietnam and provided a world checklist. Zolotuhin and Witt (2009) recorded five Andraca species from Vietnam, describing two new species, A. stueningi Zolotuhin \& Witt, 2009 and $A$. melli Zolotuhin \& Witt, 2009, and newly treating two taxa, $A$. trilochoides roepkei Bryk, 1944 and $A$. olivacea olivacens Mell, 1958, as subspecies of $A$. trilochoides Moore, 1865 and A. olivacea Matsumura, 1927 respectively. At present, the genus Andraca consists of eight species ranging from the Himalayas to Southeast Asia.

In the present paper, seven Chinese Andraca species are reviewed, including the description of one new species A. gongshanensis, sp. n. The early stages of Andraca theae (Matsumura 1909) are described in detail. A key to the seven Chinese Andraca species is provided.

\section{Materials and methods}

Specimens of the new species were collected by light trap. The types of previously described species in the Natural History Museum, London, UK (BMNH) were examined. Other materials examined in this study are preserved in SCAU and HUNAU. Morphological terminology used in descriptions follows Lemaire and Minet (1999).

\section{Taxonomy}

\section{Andraca Walker, 1865}

http://species-id.net/wiki/Andraca

Andraca Walker, 1865, List Specimens lepid. Insects Colln Br. Mus., 32: 581. (Type species: Andraca bipunctata Walker, 1865, List Specimens lepid. Insects Colln Br. Mus., 32: 582, by monotype. Type locality: Hindostan, India.) 
Pseudoeupterote Shiraki, 1911, Catalogue Insectorum Noxiorum Formosarum: 48. (Type species: Oreta theae Matsumura, 1909, Thousand Insects of Japan, 1: 582, by monotype. Type locality: Formosa (=Taiwan)). Type-species designation by monotype.

Description. Forewing weakly falcate. Ground color varying from shades of brown to sandy grey.

Male genitalia. Uncus apically single-pointed to weakly indented; gnathos with two long, basally broad, upcurved arms; valvae basally broad, sclerotized, long or medium length; aedeagus short with apex truncated, cornuti present or absent.

Female genitalia (A. bipunctata). Eighth segment curved deeply, ventral margin of ostium bursae extends posteriorly as a broad bilobed plate, ductus bursae sclerotized distal to mid-point, tapering to half width; distal half unsclerotized with slight torsion, corpus bursae lacking a signum.

Distribution. Oriental Region, S \& E Palaearctic.

Remarks. Andraca species have sometimes been described in Mustilia (e.g., Chu and Wang 1993, 1996), and misidentification has also been frequent (Chu and Wang 1993, 1996). Andraca was considered to belong to 'the Mustilia lineage' of Prismostictinae Forbes, 1955 (Holloway 1987; Minet 1994; Lemaire and Minet 1999; Holloway et al. 2001). Our own unpublished work also shows that Andraca is close to Mustilia Walker, 1865 and Mustilizans Yang, 1995, based on phylogenetic analysis of mitochondrial and nuclear DNA sequences $(C O I+18 S+28 S)$ (Wang 2010).

Sevastopulo (1938) described the fully grown larvae of the type species. The larvae are gregarious, have short hairs covering the body, and are often heavily parasitized. Pupation is in a thin cocoon of brown silk spun among leaves.

\section{Key to the Chinese Andraca species}

Apex of forewing falcate. 2

Apex of forewing not falcate .5 Uncus broad, gnathos extremely swollen medially A. bipunctata Uncus narrow, gnathos not swollen. 3 Apex of valva boot-shaped A. flavamaculata comb. rev. Apex of valva rounded or truncate 4 Apex of valva rounded, gnathos long. A. olivacea Apex of valva truncate, gnathos short A.gongshanensis sp. $\mathrm{n}$. Apex of valva bifurcate. A. theae Apex of valva rounded .6 Gnathos not swollen A. apodecta Gnathos extremely medially swollen A. melli 


\section{Andraca bipunctata Walker, 1865}

http://species-id.net/wiki/Andraca_bipunctata

Figs 1-A, 2-A

Andraca bipunctata Walker, 1865, List Specimens lepid. Insects Colln Br. Mus., 32: 582.

Type locality: Hindustan, India.

Andraca bipunctata Walker, 1862: Chu \& Wang, 1993, Sinozoologia, 10: 241.

Andraca henosa Chu \& Wang, 1993, Sinozoologia, 10: 242. Type locality: Yunnan, China.

Andraca henosa Chu \& Wang: Chu \& Wang, 1996, Fauna Sinica Insecta, 5: 55.

Description. Male (China): wingspan 42-45 mm, length of forewing 21-23 mm, antenna length 6-8 mm (Fig. 1-A). Male genitalia (Fig. 2-A): uncus broad, duck beakshaped; gnathos long, finger-shaped; vesica with a cluster of strong spinose cornuti

Female genitalia: see above under generic entry.

Material Examined. [CHINA] $2 \hat{\partial} \hat{\partial}$, western Yunnan, 2005-VI-15, Ming-Yi Tian leg.; $2 \hat{\jmath}$, Dulongjiang, Yunnan Province, 2006-VII-21, Min Wang \& XiaoLing Fan leg.; $1{ }^{\lambda} 1$, , Gongshan Mountain, Yunnan Province, 2006-VII-22 , Min Wang \& Xiao-Ling Fan leg.

Host. Camellia sinensis (Theaceae), Camellia Assamica (Theaceae), Camellia oleifera (Theaceae).

Distribution. China (Yunnan); India.

Remarks. This widely distributed species is rather variable in coloration and size. Moore (1865) described $A$. trilochoides from a brighter and grayish individual. This taxon was later synonymized with $A$. bipunctata by Hampson, ([1893]), an action that was followed by Strand (1924).

Andraca bipunctata is closely related to $A$. angulata Kishida, 1993 (Nepal and India: Sikkim), A. theae (Taiwan) and A. stueningi (Vietnam). These four species form the bipunctata group, and share the following characteristics: 1) male hindtibia with one pair of spurs; 2) two dorsally-directed projections present on subapical part of valva; 3) external surface of aedeagus partially covered with hair-like spines; 4) a cluster of strong spinose cornuti on vesica.

Larvae of $A$. bipunctata are well-known serious pests of tea trees, Camellia sinensis (Theaceae) (Banerjee 1982; Chang 1989; Chen et al. 1992; Panigrahi 1995; Ho et al. 1996; Upadhyay et al. 2001).

\section{Andraca olivacea Matsumura, 1927}

http://species-id.net/wiki/Andraca_olivacea

Figs 1-B, 2-B

Andraca olivacea Matsumura, 1927, J. Coll. Agric. Hokkaido. Univ., 19: 50. Type locality: Formosa (=Taiwan), China. 


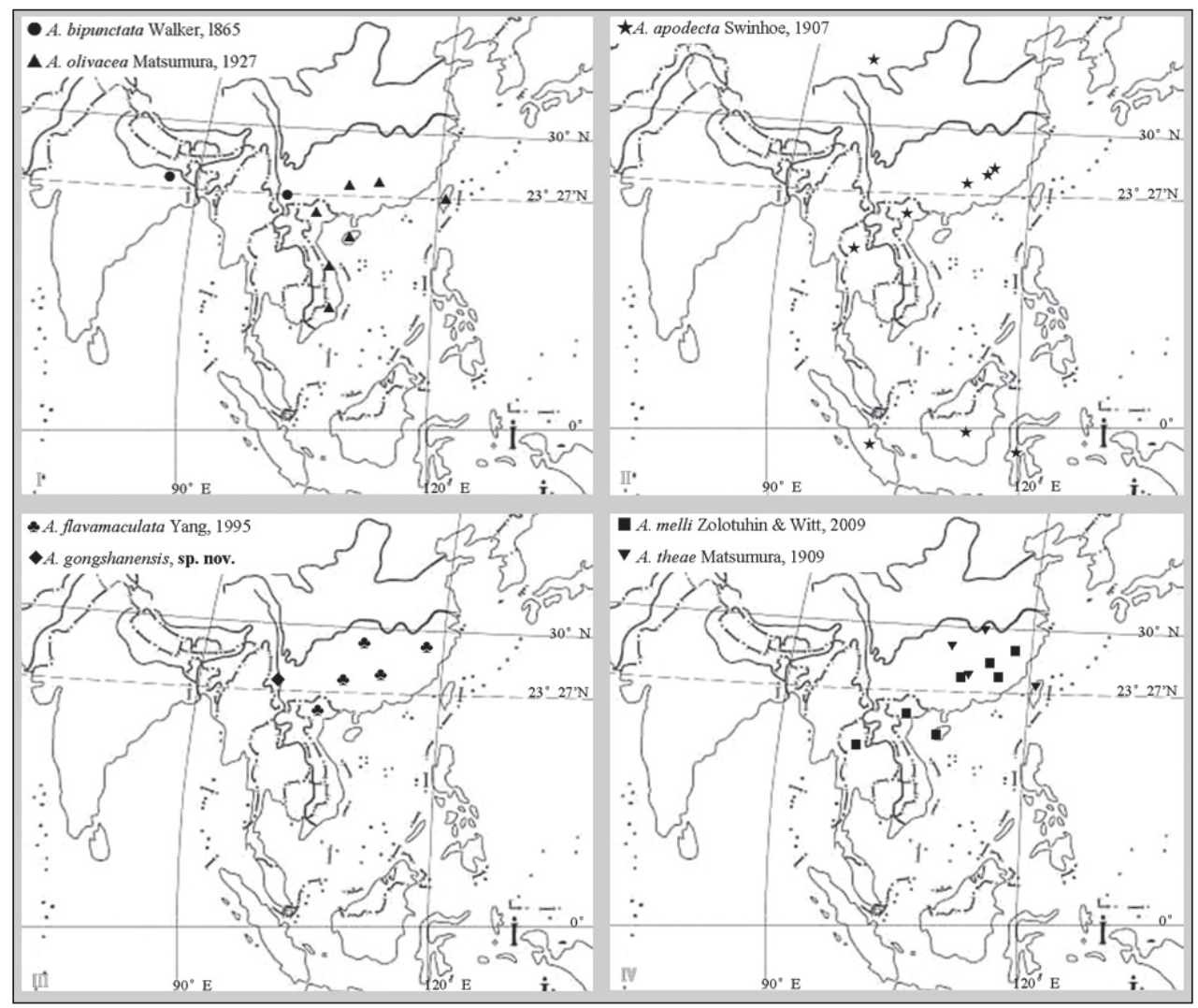

Figure I. Distributional map of Andraca spp. from China.

Andraca hedra Chu \& Wang, 1993, Sinozool., 10: 233. Type locality: Hainan, China. Andraca hedra Chu \& Wang: Chu \& Wang, 1996, Fauna Sinica Insecta, 5: 58.

Andraca olivacea: Owada et al., 2002, Spec. Bull. Jpn. Soc. Coleopterol., 5: 464; Kishida, 1992, Lepidoptera of Taiwan, 1 (2): 153.

Description. Male: wingspan 36-38 mm, length of forewing 16-20 mm, antenna length 5-7 mm (Fig. 1-B). Hindtibia with two pairs of spurs; hindwings with Rs and M1 connate. Male genitalia (Fig. 2-B): uncus thick and round; valva simple, basal half broad and terminal half narrow; distal margin of aedeagus with strong lateral spines; vesica with a cluster of spinose cornuti.

Material Examined. [CHINA] $1 \hat{\jmath}$, Shimentai Provincial Nature Reserve, Yingde City, Guangdong Province, 2001-VII-24, Min Wang \& Guo-Hua Huang leg.; 1 ग, same data but 2001-IX-22; 3 $\widehat{\jmath}$, same data but 2002-VI-11, Guo-Hua Huang leg.; $1 \lesssim$, Nanling National Nature Reserve, Ruyuan City, Guangdong Province, 2002-VII23, Guo-Hua Huang leg.; $4 \hat{\sigma} \widehat{\jmath}$, same data but 2003-III-29 31; $1 \hat{\jmath}$, same data but

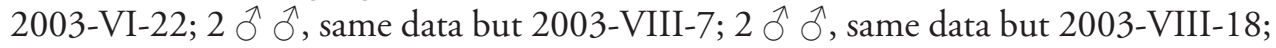

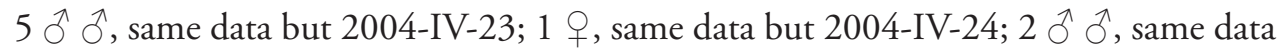


but 2006-IX-18, Liu-Sheng Chen leg.; $1 \hat{\jmath}$, same data but 2008-VI-7, Min Wang leg.; $1 \hat{\partial}$, same data but 2008-VI-7; $1 \hat{\partial}$, same data but 2009-IV-1, Hou-Shuai Wang leg.; $1 \hat{\delta}$, same data but 2009-VIII-10; $1 \hat{\delta}$, same data but 2009-IV-1; $1 \hat{\delta}$, same data but

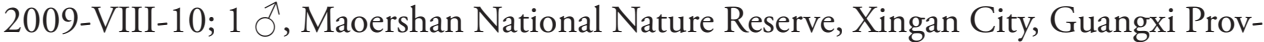
ince, 2003-III-3, Min Wang \& Guo-Hua Huang leg.; $6 \widehat{\partial} \widehat{\delta}$, Jianfengling National Nature Reserve, Ledong City, Hainan Province, 2003-XI-29 -31, Guo-Hua Huang \& Min Wang leg.; $1 \hat{O}$, same data but 2007-X-23, Min Wang leg.

Host. Ficus concinna var. pusillifolia (Moraceae).

Distribution. China (Taiwan, Guangdong, Guangxi, Hainan); Vietnam.

Remarks. Wang (1995) provide a fine color illustration of a fresh living male. Owada et al. (2002) considered Andraca olivacens from Fukien (= Fujian) to the synonym of A. olivacea, whereas Zolotuhin and Witt (2009) treated it as a subspecies thereof. We do not comment further on which of these two alternatives may be the most appropriate status for this taxon because we have not seen the types of $A$. olivacens.

\section{Andraca apodecta Swinhoe, 1907}

http://species-id.net/wiki/Andraca_apodecta

Figs 1-C, 2-C

Andraca apodecta Swinhoe, 1907, Ann. Mag. nat. Hist., 19 (7): 49. Type locality: Sumatra, Indonesia.

Andraca apodecta Swinhoe: Holloway, 1976, Malayan Nature Society: 85; Zolotuhin \& Witt, 2009, Entomofauna, 261.

Description. Male: wingspan 37-39 mm, length of forewing $15-18 \mathrm{~mm}$, antenna length 6-8 mm (Fig. 1-C). Head covered with reddish-brown hairs; forewing with black discal spot, smooth outer margin and apically not falcate. Male genitalia (Fig. 2-C): uncus triangular, apical half truncate; valva with two subapical, dorsally-directed projections; aedeagus short, curved slightly without cornuti, external surface without hair-like spines.

Material Examined. [CHINA] $2 \widehat{\partial}$, Jinzhongshan Mountain, Longlin City, Guangxi Province, 2007-VII-31, Liu-Sheng Chen leg..

Host. Unknown.

Distribution. China (Guangxi, Yunnan, Fujian, Shaanxi), Vietnam, Thailand (Chiang Mai, Nan), Indonesia (Sumatra, Borneo, Sulawesi).

Remarks. The species was first recorded from China (Yunnan, Fujian, Shaanxi) by Zolotuhin and Witt (2009) and is here recorded from Guangxi for the first time. 


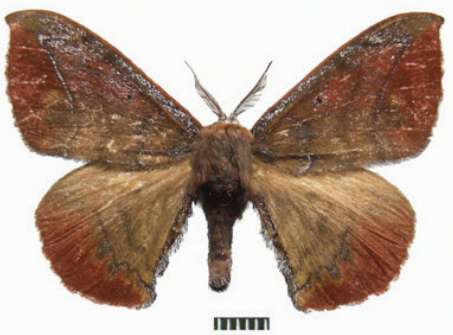

A

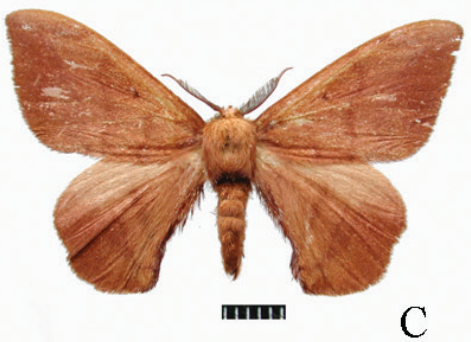

$\mathrm{C}$
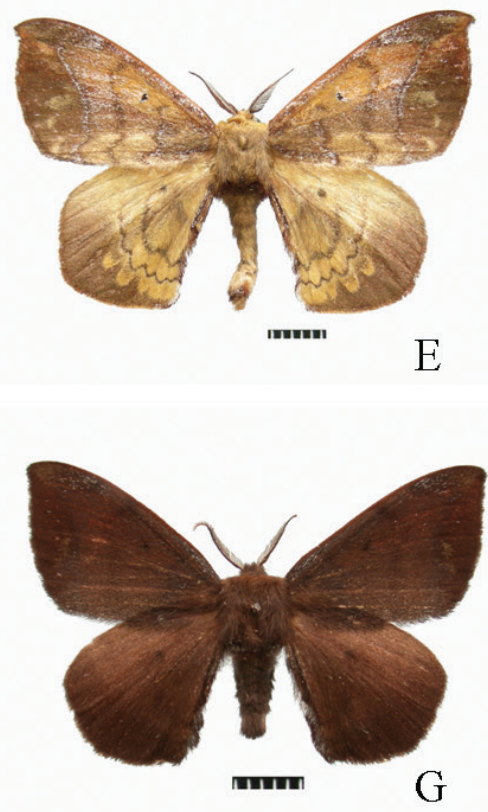

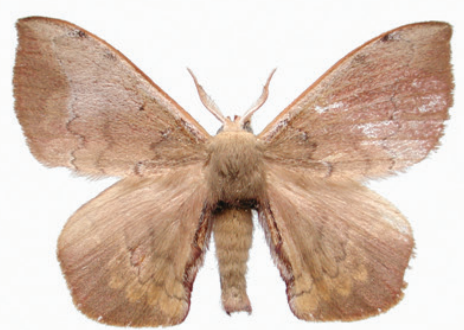

$\mathrm{B}$
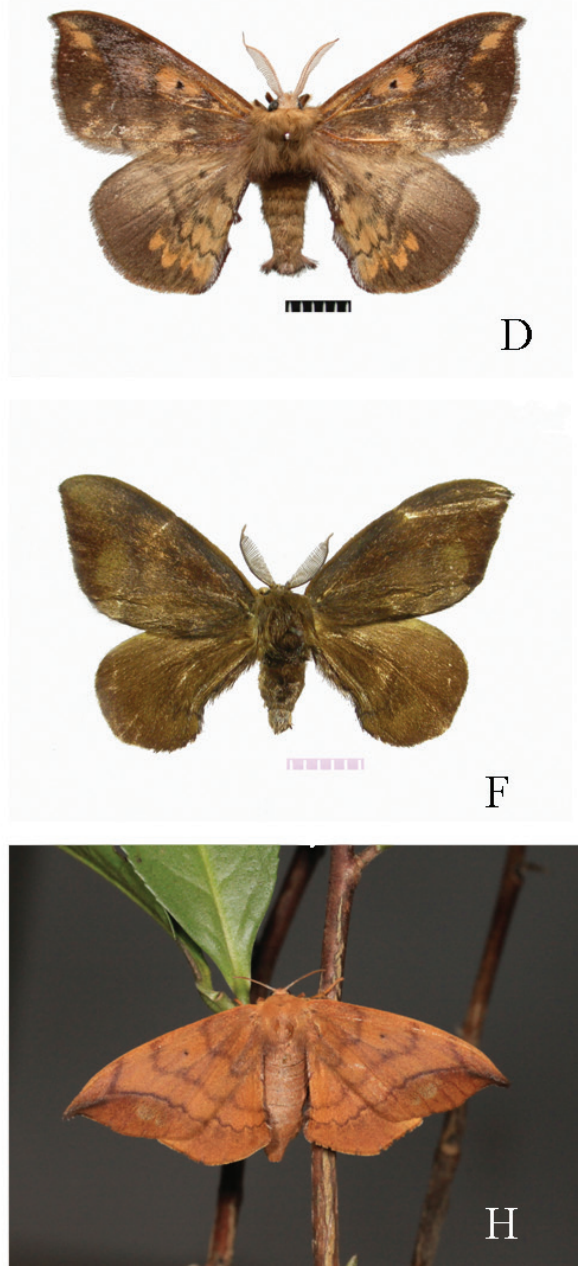

Figure 2. Adult male Andraca spp. A A. bipunctata Walker, 1865 from Yunnan B A. olivacea Matsumura, 1927 from Guangdong C A. apodecta Swinhoe, 1907 from Guangxi D A. flavamaculata Yang, 1995 from Guangdong E A. gongshanensis, sp. n., Holotype, from Yunnan F A. melli Zolotuhin \& Witt, 2009 from Guangdong $\mathbf{G} A$. theae Matsumura, 1909 from Hunan Province H $A$. theae Matsumura, 1909 from Hunan Province (in field). 
Andraca flavamaculata Yang, 1995, comb. rev. http://species-id.net/wiki/Andraca_flavamaculata Figs 1-D, 2-D

Andraca flavamaculata Yang, 1995, Insects of Baishanzu Mountain, Eastern China: 354. Type locality: Zhejiang, China.

Andraca nabesan Kishida \& Owada, 2002, Spec. Bull. Jpn. Soc. Coleopterol., (5): 464; Huang \& Wang, 2004, Entomotaxonomia, 26(1): 47. Type locality: Cao Bang, Vietnam.

Description. Male: wingspan 40-44 mm, length of forewing 20-22 mm, antenna length 6-7 mm (Fig. 1-D). Body stout. Forewing apex falcate; outer edge smooth and straight; tornus almost rectangular. Male genitalia (Fig. 2-D): uncus long with apex finger-shaped; tegumen broad with numerous long setae; valvae basally broad, strongly sclerotized, apex of valva boot-shaped; sacculus broad, with a strong dorsal spike; saccus short and narrow; aedeagus short but strong and straight, distally with a large number of spines.

Material Examined. [CHINA] $2 \widehat{\partial} \widehat{\partial}$, Nanling National Nature Reserve, Ruyuan City, Guangdong Province, 2002-III-15, Guo-Hua Huang leg.; $2 ~ \lesssim ぇ$, same data but

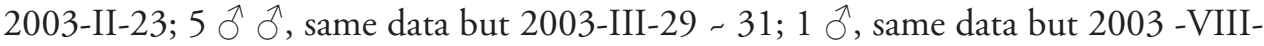

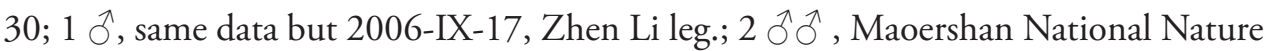
Reserve, Xingan City, Guangxi Province, 2003-III-03, Min Wang \& Guo-Hua Huang leg.; 3 $\widehat{\partial}$, Mangshan Nature Reserve, Yizhang City, Hunan Province, 2003-III-31, Guo-Hua Huang leg.; 1 ${ }^{\lambda}$, Jiuwandashan National Nature Reserve, Guangxi Province, 2003-VII-30, Guo-Hua Huang leg.

Host. Unknown.

Distribution. China (Zhejiang, Hunan, Guangdong, Guangxi); Vietnam.

Remarks. Yang (1995) described the species from Zhejiang, China. The species is similar to Andraca olivacea but can be distinguished by the following characters: aedeagus straight; gnathos not prominent. Zolotuhin and Witt (2009) synonymized A. nabesan Kishida \& Owada, 2002 with $A$. flavamaculata, which they also transferred to Pseudandraca species based on features of the genitalia. We accept the synonymy but do not agree with the generic transfer, because we do not consider that the diagnostic feature of Pseudandraca given by Miyata (1970), a valva with a "long distinct projection" is present in A. flavamaculata. We therefore transfer A. flavamaculata comb. rev. back to Andraca.

\section{Andraca gongshanensis sp. $\mathrm{n}$.}

urn:lsid:zoobank.org:act:E5DD5FB7-554B-48A6-9EF3-65F1699E9897

http://species-id.net/wiki/Andraca_gongshanensis

Figs 1-E, 2-E

Description. Male: wingspan 46-48 mm, length of forewing 22-24 mm, antenna length 5-8 mm (Fig. 1-E). Antenna bipectinate except apex. Wings ground color 
dark brow with dark brow fasciae and reddish-yellow patterns, which is consisting of antemedian, discocellar, postmedian fascia, and reddish-yellow patterns nearly placed on the wholly wings but termen. Forewing apex falcate; outer edge smooth and straight; tornus almost rectangular. Hindwing with anal margin straight; outer margin angled at vein $M_{3}$, straight above and below this.

Male genitalia (Fig. 2-E): uncus long with wedge-shaped apex; tegumen broad; gnathos very well developed, arms upcurved; valvae basally broad with many long setae, strongly sclerotized, caudally constricted to a spatulate apex; sacculus broad, without a dorsal spike; aedeagus short but strong and straight, distally with a large number of spines.

Holotype. ${ }^{\lambda}$, Gongshan Mountain, Yunnan Province, China, 2006-VII-22, Min Wang \& Xiao-Ling Fan leg., deposited in Department of Entomology, SCAU; Paratypes, $2 \hat{\jmath}$, same data as holotype but 2006-VII-21.; $1 \hat{\jmath}$, same data as holotype but 2006-VII-23; deposited in Institute of Entomology, HUNAU.

Host. Unknown.

Distribution. China (Yunnan).

Etymology. The specific epithet refers to the type locality (Gongshan Mountain, China).

Remarks. This new species is very similar to A. flavamaculata, but can be distinguished by the following characters of the male genitalia: A. gongshanensis, sp. n. with uncus apex wedge-shaped, apex of valva constricted and truncate, sacculus without a strong dorsal spike. And A. flavamaculata with uncus apex finger-shaped, apex of valva boot-shaped; sacculus broad, with a strong dorsal spike.

Externally, A. gongshanensis is paler than A. flavamaculata.

Andraca melli Zolotuhin \& Witt, 2009

http://species-id.net/wiki/Andraca_melli

Figs 1-F, 2-F

Andraca melli Zolotuhin \& Witt, 2009, Entomofauna, Suppl. 16: 262. Type locality: Guangdong, China.

Description. Male: wingspan 37-39 $\mathrm{mm}$, length of forewing 15-18 $\mathrm{mm}$, antenna length 5-7 mm (Fig. 1-F). Antenna bipectinate except apex. Head thinly covered with browngreen hairs. Forewing: apically bluntly pointed; outer edge smooth and straight, tornus nearly rectangular. Hindwings distinctly angled at vein $\mathrm{M}_{3}$, straight above and below this.

Male genitalia (Fig. 2-F): uncus bluntly triangular with long hairs; tegumen broad; gnathos with two extremely medially swollen arms; valvae flattened, strongly sclerotized, apex narrower and truncate with a dorsally directed projection from the middle; saccus short and broad; aedeagus short, strongly curved, with a compact group of long, thick needle-shaped cornuti on dorsal surface. 
Material Examined. [CHINA] $2 \hat{\jmath}$, Nanling National Nature Reserve, Ruyuan City, Guangdong Province, 2007-VI-23, Liu-Sheng Chen collected larvae and reared to adult.

Host. Camellia sinensis (Theaceae), Camellia oleifera (Theaceae), Fraxinus pennsylvanica (Oleaceae) and Ternstroemia japonica (Ternstroemiaceae), Pentaphylax euryoides Gardn. \& Champ. (Pentaphylacaceae) (new host record).

Distribution. China (Zhejiang, Jiangxi, Fujian, Guangdong, Hainan); Vietnam; Thailand.

Remarks. A. melli was first described by Zolotuhin and Witt (2009), who also reported on the biology of this species.

\section{Andraca theae Matsumura, 1909}

http://species-id.net/wiki/Andraca_theae

Figs 1-G, 1-H, 2-G, 2-H

Oreta theae Matsumura, 1909, Thousand Insects of Japan, 1: 86. Type locality: Formosa (= Taiwan), China.

Description. Male: wingspan 35-37 mm, length of forewing $17-19 \mathrm{~mm}$, antenna length 6-7 mm (Figs 1-G, 1-H). Head densely covered with dark brown hairs; antenna bipectinate except apex. Forewing apex inconspicuously falcate, exterior margin straight. Forewing and hindwing each with a dark discal spot.

Male genitalia (Fig. 2-G): uncus triangular with apex narrowly spatulate; tegumen broad; gnathos elongate, medially inflated; saccus short and broad; valvae bifurcate apically; dorsal margin with a subapical hump; aedeagus bowed with dense apical spines.

Material Examined. [CHINA] 1 , Nanling National Nature Reserve, Ruyuan City, Guangdong Province, 2003-III-29, Guo-Hua Huang leg.; $1 \hat{\delta}$, same data to the former, except 2003-VIII-12, Guo-Hua Huang \& De-Yu Xin leg.; $2 \lesssim \precsim$, Taibei City,

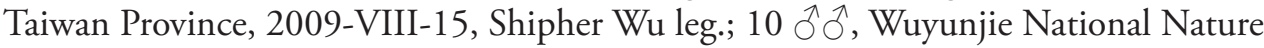
Reserve, Taoyuan City, Hunan Province, 2010-VII-2, collected the larvae in the field by Mr. Hong-Chun Zhou, got the adults from the larvae bred in the entomological

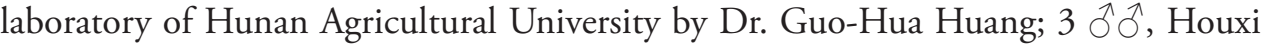
Town, Huangshan City, Anhui Province, 2010-VI-28, the adults from the larvae collected in the field and bred in laboratory by Dr. Guo-Hua Huang.

Host. Camellia sinensis (Theaceae).

Biology. This species is widely distributed in Taiwan and Southern China. The larvae were found on Camellia sinensis in Hunan Province; photographs of the early stages were taken in June to August, 2010 (Figs 3-A to 3-H).

Distribution. China (Taiwan, Guangdong, Hunan, Anhui).

Remarks. The species is easily separated from its congeners by the apically bifurcate valvae. 

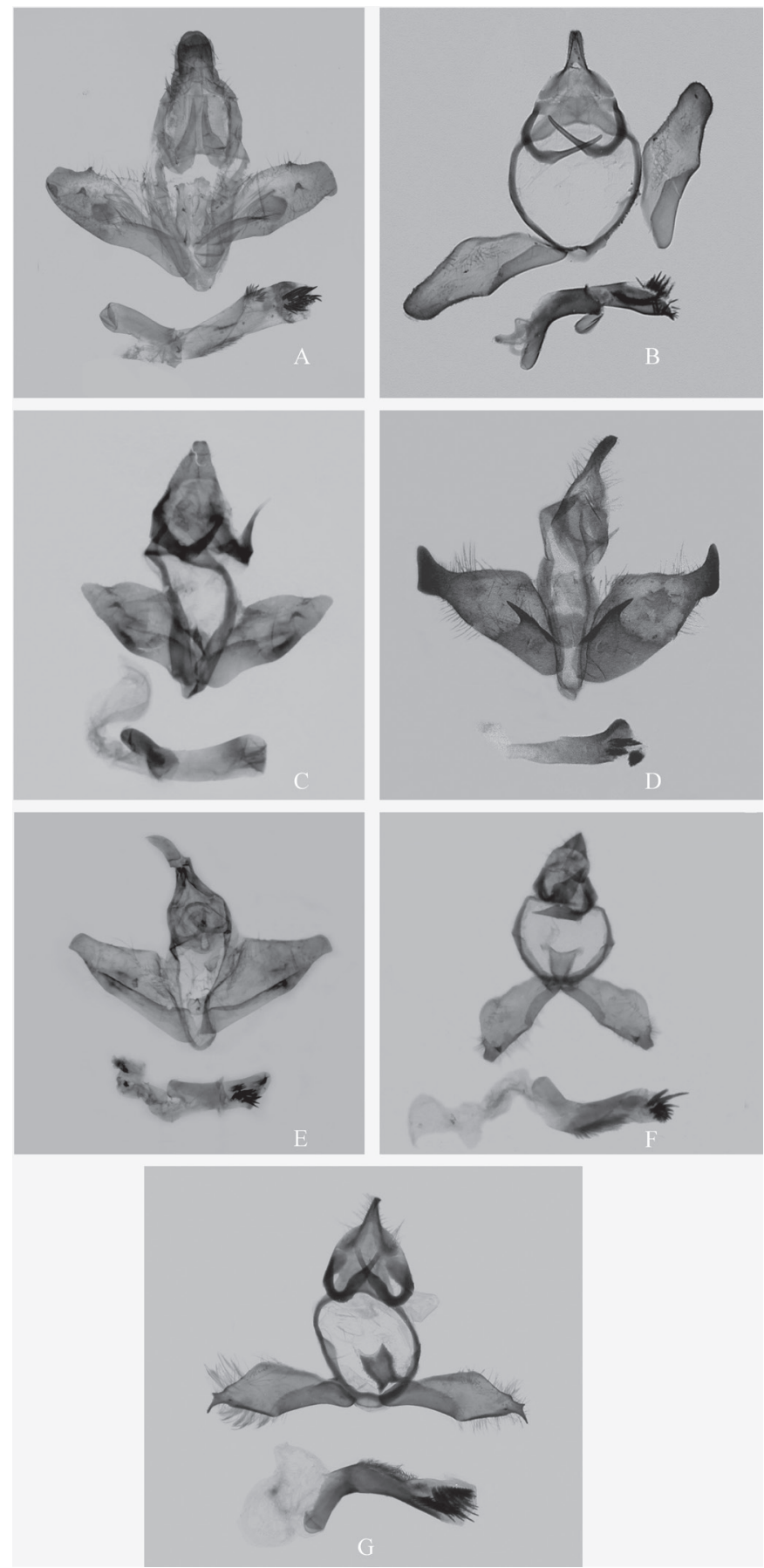

Figure 3. Male genitalia of Chinese Andraca spp. A A. bipunctata Walker, 1865 from Yunnan B A. olivacea Matsumura, 1927 from Guangdong C $A$. apodecta Swinhoe, 1907 from Guangxi D A. flavamaculata Yang, 1995 from Guangdong E A. gongshanensis, sp. n., Holotype, from Yunnan F A. melli Zolotuhin \& Witt, 2009 from Guangdong $\mathbf{G}$ A. theae Matsumura, 1909 from Hunan Province. 

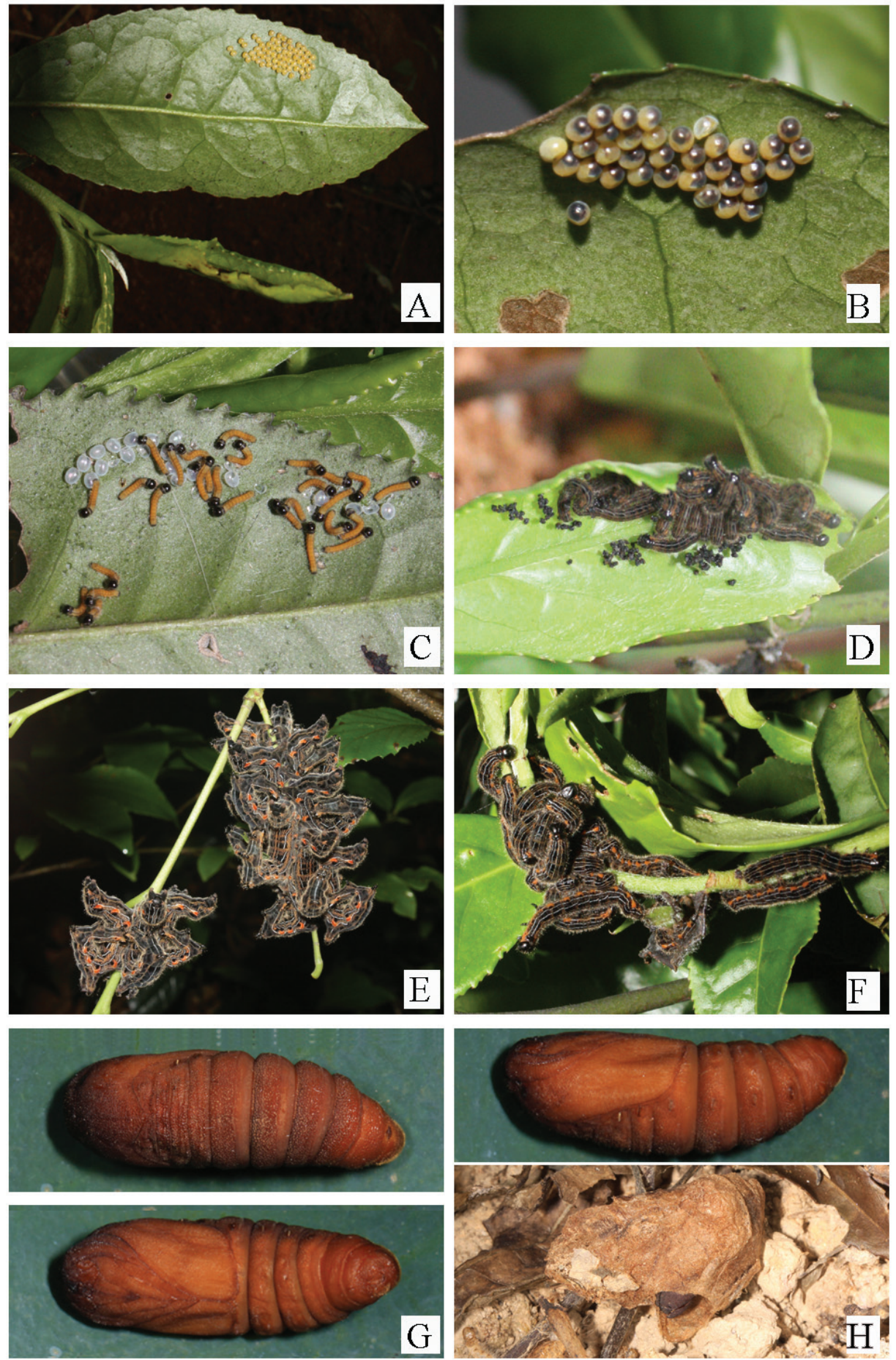

Figure 4. The early stages of Andraca theae Matsumura, 1909 from Hunan Province A-B Eggs C First larvae D Third larvae E-F Final larvae G-H Pupa and cocoon. 


\section{Acknowledgments}

We would like to thank Mr. Shipher Wu (Taiwan National University, Taibei), Dr. Guo-Hua Huang and Mr. Hong-Chun Zhou (HUNAU), Dr. Liu-Sheng Chen (Shihezi University, China), Mr. De-Yu Xin and Hou-Shuai Wang (SCAU) for collecting specimens in the field. Many thanks to Dr. Mamoru Owada (National Museum of Nature and Science, Tokyo, Japan), Vadim V. Zolotuhin (State Pedagogical University of Ulyanovsk, Russia) and Andreas Zwick (State Museum of Natural History Stuttgart, Germany) for their kind suggestions and help with the manuscript. We are grateful to Prof. James Mallet (University College London, UK) kindly corrected the language of the manuscript. We thank the late Dr. G. S. Robinson and Mr. K. Tuck (The Natural History Museum, UK) for checking the types. This work was supported by Scientific Research Fund of Hunan Provincial Education Department (10B048) and the Natural Science Foundation of China (no. 31100482).

\section{References}

Banerjee B (1982) A strategy for the control of Andraca bipunctata Walker on tea. Crop Protection 1 (1): 115-119.

Bryk F (1945) Entomological results from the Swedish expedition 1934 to Burma and British India. Lepidoptera: Saturniidae, Bombycidae, Eupterotidae, Uraniidae, Epiplemidae und Sphingidae. Arkiv Zoology 35A (8): 1-55, pls. 1-6.

Chang HG (1989) Effects of temperature on development of the egg stage of the tea bunch caterpillar Andraca bipunctata Walker. Journal of Anhui Agricultural College 16 (2): 137-140.

Chen JX, Ding YG \& Xu DJ (1992) Studies on a granulosis virus of the tea bunch caterpillar Andraca bipunctata [LEP.: Bombycidae] and its utilization. Chinese Journal of Biological Control 8 (2): 72-76.

Chu HF, Wang LY (1993) Saturniidae of China. Bulletin of Zoology. Science Press. Beijing, 10: 211-238.

Chu HF, Wang LY (1996) Fauna Sinica Insecta V, Lepidoptera: Bombycidae. Beijing: Science Press. 1996: 24pp.

Davis DR (1992) Bombycidae. In: Heppner JB, Inoue H (Eds) Lepidoptera of Taiwan 1 (2): Checklist. Scientific Publishers, Gainesville/Washington/Hamburg/Lima/Taipei/ Tokyo, 153-154.

Hampson GF (1893) Moths, 1. In: Fauna of British India, Including Ceylon and Burma. Taylor \& Francis, London. xxiii +527 pp.

Ho HY, Tao YT, Tsai RS, Wu YL, Tseng HK, Chow YS (1996) Isolation, identification, and synthesis of sex pheromone components of female tea cluster caterpillar Andraca bipunctata walker (Lepidoptera: Bombycidae) in Taiwan. Journal of Chemical Ecology 22 (2): 271-285.

Holloway JD (1976) Moths of Borneo with Special Reference to Mount Kinabalu. Malayan Nature Society, Kuala Lumpur. [viii] + 264 pp. 
Holloway JD (1987) The moths of Borneo (III). Kuala Lumpur: Malaysian Nature Society, 74-90.

Holloway JD, Kibby G, Peggie D (2001) Fauna Malesiana Handbook, 3. The Families of Malesian Moths and Butterflies. Brill, Leiden/Boston/Köln. xi +455 pp.

Kishida Y (1993) Bombycidae. In: Haruta T (Ed) Moths of Nepal, Part 2. Tinea 13 (Supplement 3): 143-145, pl. 57.

Lemaire C, Minet J (1999) The Bombycoidea and their relatives. In: Kristensen NP (Ed) Lepidoptera, Moths and Butterflies. 1. Evolution, systematics and biogeography. Handbook of Zoology 4(35), Walter de Gruyter, Berlin \& New York, 321-354.

Minet J (1994) The Bombycoidea-Phylogeny and higher classification (Lepidoptera, Glossata). Entomologica Scandinavica 25 (1): 63-88.

Miyata T (1970) A generic revision of the Japanese Bombycidae, with description of a new genus (Lepidoptera). Tinea 8: 190-199.

Moore F (1865) On the lepidopterous insects of Bengal. Proceedings of the Zoological Society of London, 1865: 755-823, pls. 41-43.

Owada M, Kishida Y, Thinh TH, Jinbo U (2002) Moths of the genus Andraca (Lepidoptera, Bombycidae, Prismostictinae) from Vietnam. Special Bulletin of the Japanese Society of Coleopterology (5): 461-472.

Panigrahi A (1995) Fungus Cordyceps militaris (Fries) link infestation in the pupa of the tea pest Andraca bipunctata Walker. Environment and Ecology 13 (4): 942-946.

Sevastopulo DG (1938) Early stages of Indian Lepidoptera (18). Journal of Bombay Natural History Society 35: 40-46.

Strand E (1924) Bombycidae. In: Seitz A (Ed) Macrolepidoptera of the World 10: 436-442, 457.

Upadhyay RK, Mukerji KG, Chamola BP (Editor) (2001) Biocontrol Potential and its Exploitation in Sustainable Agriculture: Volume 2: Insect Pests. Springer Publisher, 162-163.

Walker F (1865) List of the specimens of lepidopterous insects in the collection of the British Museum 32, (suppl. to part 2), London, 323-706.

Wang HY (1995) Guide Book to Insects in Taiwan: Bombycidae, Thyatiridae, Limacodidae, Lasiocampidae and Sphingidae. Taipei: Shuhsing Publishing, 10-11.

Wang X (2010) Studies on the Systematics of Bombycidae from China (Lepidoptera: Bombycoidea). Unpublished PhD Thesis (South China Agricultural University, China.)

Yang JK (1995) Lepidoptera: Bombycidae. In: Wu H (Ed) East Hill Zukun hundred insects. China Forestry Publishing House, Beijing, 353-358.

Zolotuhin V, Witt TJ (2009) The Bombycidae of Vietnam. Entomofauna, (Suppl. 16): 231272.

Zwick A, Regier JC, Mitter C, Cummings MP (2011) Increased gene sampling yields robust support for higher-level clades within Bombycoidea (Lepidoptera). Systematic Entomology, 36: 31-43. 